\title{
Experimental obtaining of polarization ghost images by ghost polarimetry
}

\author{
Dmitry Agapov, Sergei Magnitskiy, and Anatoly Chirkin \\ Faculty of Physics and International Laser Center of Lomonosov Moscow State University, 1119991 \\ Moscow, Russia
}

\begin{abstract}
The paper presents the experimental implementation of a new version of the ghost image method - ghost polarimetry in unpolarized light. Like conventional polarimetry, ghost polarimetry is used to study the polarization properties of objects. Our proposed version of this technique is applied to objects whose influence on the polarization state of light is determined by linear dichroism. The restoration of the polarization properties of the object was carried out in the framework of the theory that we constructed on the basis of the apparatus of Jones matrices.
\end{abstract}

Polarimetry is a powerful modern method for studying the polarization properties of objects. Polarimetry is based on monitoring the change in the polarization state of optical radiation after interaction with the object. However, transmitted or reflected radiation is not always available for scanning, which imposes restrictions on the spatial resolution of this method. The ghost imaging (GI) technique can be used to overcome these limitations.

The method of GI [1] is based on the principles that form the basis of the intensity interferometer. The process of reconstructing ghost images consists in measuring the correlation function between the total radiation intensity after interacting with an object and the intensity of a light beam that does not interact with an object at all. The combination of polarimetry and theeghost imaging method was called ghost polarimetry [2].

The effect of an object on the polarization state of optical radiation can be described by Jones matrices. In [3], we showed the relationship of the elements of the Jones matrix of the object with the correlation functions of optical radiation, which should be depolarized and have thermal statistics. To obtain an image, the spatial intensity distributions of two orthogonal polarization components $(x, y)$ are measured in a reference beam. In particular, for objects with linear dichroism, and therefore exhibiting the properties of a polarizer, a functional relationship was obtained between the angle $\theta$ determining the direction of the polarizer axis and the correlation functions:

$$
|\operatorname{tg}(\theta(i, j))|=\sqrt{\frac{G_{y}(i, j)}{G_{x}(i, j)}}
$$

where $G_{y, x}(\boldsymbol{i}, \boldsymbol{j})$ - the correlation function of two beams, $x$ and $y$ are the indices which denote the direction of polarization; $\boldsymbol{i}, \boldsymbol{j}$ are coordinates of the radius of the vector defining the point in the plane of the object. 
Based on the proposed theory, an experimental technique was created. It is important that in the the object channel there are no polarizing elements. The images obtained in the proposed ghost polarimetry scheme were called polarizing ghost images. Such ghost images contain additional information about the object relative to ordinary ghost images, namely, information on the distribution of its polarization properties. Figure (1) shows the experimentally obtained polarization image of a slit with an applied polarizer.

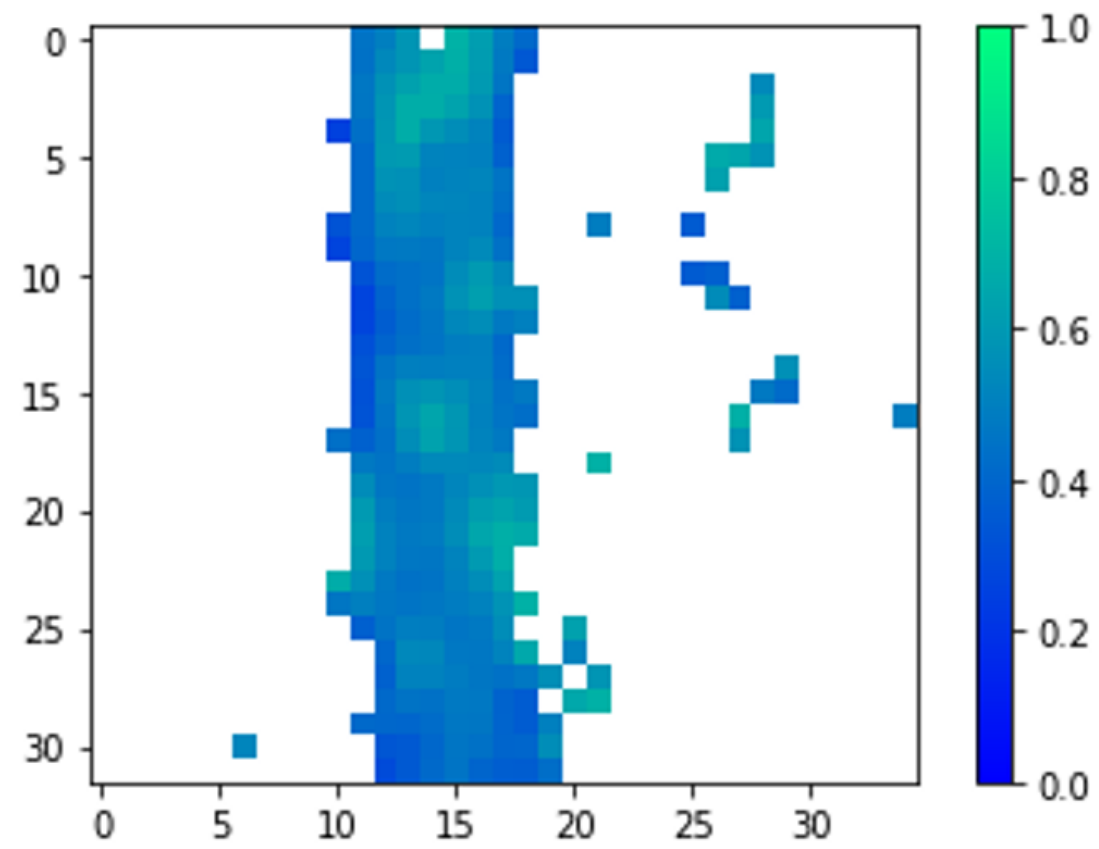

Fig. 1. Polarization ghost image of a slit with superimposed polarizer.

In this case, the image contains information about the spatial distribution $|\operatorname{tg}(\theta(i, j))|$. A slit with a linear polarizer superimposed on it is used as an object of study. The average value of $|\operatorname{tg}(\theta(i, j))|$ along the slit profile was 0.536 , which corresponds to an angle $\pm 28.2^{0}$ in relation to the axis $x$. The angle of the polarizer axis was $30^{\circ}$ the average value relative to the axis $x$. The sign is selected based on additional measurements in a rotated polarization basis.

Thus, the previously developed theory and experimentally obtained polarization FIs allow us to conclude that ghost polarimetry, along with traditional polarimetry, can be used to study the polarization properties of objects.

The work is partially supported by the RFBR grant No.18-02-00-849.

\section{References}

1. Belinsky A.V., Klyshko D.N. // JETP. 1994. V. 105, No 3, p. 487.

2. Kellock H., Setälä T., Friberg A. T., \& Shirai T. // Journal of Optics 16.5 (2014): 055702.

3. A.S. Chirkin, P.P. Gostev, D.P. Agapov, S.A. Magnitskiy, Laser Physics Letters, 15(11) (2018) 